\title{
DE PERSONAS DESECHABLES A "TRABAJADORES ESENCIALES": algunos apuntes sobre los latino/as y el COVID-19 en los Estados Unidos
}

\author{
FROM DISPOSABLE PEOPLE TO "ESSENTIAL WORKERS": some notes on Latinos \\ and COVID-19s in the United States
}

\author{
DE PESSOAS DESCARTÁVEIS À “TRABALHADORES ESSENCIAIS”: alguns \\ apontamentos sobre os latinos/as e a COVID-19 nos Estados Unidos
}

Suzanne Oboler

P.h.D.

Professor, Latin American and Latinx Studies and Faculty Steering Committee, Human Rights Minor John Jay College of Criminal Justice/CUNY soboler@jjay.cuny.edu

Estados Unidos

\section{Resumen}

¿Cómo se determina si una sociedad es justa? Hay los que dicen que se verifica a través del trato que la sociedad proporciona a sus miembros más vulnerables. La devastación que el COVID-19 sigue dejando en los Estados Unidos y en varios otros países nos lleva a indagar cómo están los más vulnerables entre nosotros... Escogí enfocar a la comunidad latina en los Estados Unidos, porque es una comunidad que incluye a muchos miembros que hacen parte de los sectores más vulnerables de la sociedad estadounidense. Paradójicamente, es una comunidad fuerte y grande y, de hecho, hoy es el grupo minoritario más grande del país. Actualmente hay casi 60 millones de latino/as, que representan el $18 \%$ de la población. Es también importante subrayar que aproximadamente el $65 \%$ de los latino/as, o sea más de la mitad de esta comunidad, nacieron en los Estados Unidos y, por lo tanto, son ciudadanos americanos. Es más, a ellos se suman otros $15 \%$ entre los latino/as que se han convertido en ciudadanos de este país. Entonces, a pesar de todos los esfuerzos del gobierno por pintar a la comunidad latina como si fuese compuesta enteramente de inmigrantes, - la realidad es que, en esta sociedad, los latino/as son en su mayoría ciudadanos estadounidenses. Es más, es una comunidad muy heterogénea: entre ellos se encuentra una diversidad de etnias, razas, generaciones, clases sociales, idiomas, identidades sexuales, etc.

Palabras clave: personas desechables, trabajadores esenciales, COVID-19, Estados Unidos 


\title{
Resumo
}

Como você determina se uma sociedade é justa? Há quem diga que é verificado através do tratamento que a sociedade proporciona aos seus membros mais vulneráveis. A devastação que o COVID-19 continua a deixar nos Estados Unidos e em vários outros países nos leva a investigar como os mais vulneráveis entre nós são... Escolhi focar a comunidade latina nos Estados Unidos, porque é uma comunidade que inclui muitos membros que fazem parte dos setores mais vulneráveis da sociedade americana. Paradoxalmente, é uma comunidade forte e grande e, de fato, hoje é o maior grupo minoritário do país. Atualmente, há quase 60 milhões de latinos, representando $18 \%$ da população. Também é importante enfatizar que aproximadamente $65 \%$ dos latinos, ou mais da metade desta comunidade, nasceram nos Estados Unidos e, portanto, são cidadãos americanos. Mais do que isso, eles são adicionados outros $15 \%$ entre os latinos que se tornaram cidadãos deste país. Então, apesar de todos os esforços do governo para pintar a comunidade latina como se fosse composta inteiramente de imigrantes, a realidade é que, nesta sociedade, os latinos são principalmente cidadãos americanos. Mais importante, é uma comunidade muito heterogênea: entre elas está uma diversidade de etnias, raças, gerações, classes sociais, línguas, identidades sexuais, etc.

Palavras chave: pessoas descartáveis, trabalhadores essenciais, COVID-19, Estados Unidos.

\begin{abstract}
How do you determine whether a society is fair? There are those who say that it is verified through the treatment that society provides to its most vulnerable members. The devastation that COVID-19 continues to leave in the United States and in several other countries leads us to look into how the most vulnerable among us are... I chose to focus the Latino community in the United States, because it is a community that includes many members who are part of the most vulnerable sectors of American society. Paradoxically, it is a strong and large community and, in fact, today it is the largest minority group in the country. There are now almost 60 million Latinos, representing $18 \%$ of the population. It is also important to emphasize that approximately $65 \%$ of Latinos, or more than half of this community, were born in the United States and are therefore American citizens. More than that, they are added another $15 \%$ among Latinos who have become citizens of this country. So, despite all the government's efforts to paint the Latino community as if it were made up entirely of immigrants, - the reality is that, in this society, Latinos are mostly American citizens. More importantly, it is a very heterogeneous community: among them is a diversity of ethnicities, races, generations, social classes, languages, sexual identities, etc.
\end{abstract}

Palavras chave: disposable people, essential workers, COVID-19, USA. 


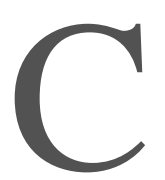

ómo se determina si una sociedad es justa? Hay los que dicen que se verifica a través del trato que la sociedad proporciona a sus miembros más vulnerables. La devastación que el COVID-19 sigue dejando en los Estados Unidos y en varios otros países nos lleva a indagar cómo están los más vulnerables entre nosotros... Escogí enfocar a la comunidad latina en los Estados Unidos, porque es una comunidad que incluye a muchos miembros que hacen parte de los sectores más vulnerables de la sociedad estadounidense. Paradójicamente, es una comunidad fuerte $\mathrm{y}$ grande $\mathrm{y}$, de hecho, hoy es el grupo minoritario más grande del país. Actualmente hay casi 60 millones de latino/as, que representan el $18 \%$ de la población. Es también importante subrayar que aproximadamente el $65 \%$ de los latino/as, o sea más de la mitad de esta comunidad, nacieron en los Estados Unidos $\mathrm{y}$, por lo tanto, son ciudadanos americanos. Es más, a ellos se suman otros $15 \%$ entre los latino/as que se han convertido en ciudadanos de este país. Entonces, a pesar de todos los esfuerzos del gobierno por pintar a la comunidad latina como si fuese compuesta enteramente de inmigrantes, -la realidad es que, en esta sociedad, los latino/as son en su mayoría ciudadanos

Suzanne Oboler. Extraños Desechables: raza e inmigración en la era de la globalización" InterDisciplina: 2(4) Sept/Dec, 2014; pp 75-96 estadounidenses. Es más, es una comunidad muy heterogénea: entre ellos se encuentra una diversidad de etnias, razas, generaciones, clases sociales, idiomas, identidades sexuales, etc.

La comunidad se volvió más vulnerable a partir de julio del 2015, cuando Trump, en ese entonces candidato a la presidencia del país, comenzó su campaña con insultos contra los "mexicanos." Los ataques del presidente Trump aceleraron una tendencia que ya venía desde los comienzos de esa década, cuando el término "mexicano" comenzó a volverse un eufemismo para referirse a todos los latino/as en los Estados Unidos. Asimismo, hoy en día es sinónimo de "inmigrante." O sea, para muchos estadounidenses, todos los latino/as son mexicanos, todos los mexicanos son inmigrantes, y, en este sentido, se les ve no tanto como ciudadanos estadounidenses, sino más bien como extranjeros, recién llegados, gente que no pertenece a la sociedad estadounidense. ${ }^{1}$

Durante esta época del COVID-19 esta realidad ha creado una paradoja: a pesar de que a menudo se les perciba como población recién llegada, y que no pertenezcan al país, sin embargo, hoy, en su mayoría, los latino/as también son definidos como "trabajadores esenciales" en la 
sociedad estadounidense. ¿Quiénes son "trabajadores esenciales"? A nivel más básico, esta terminología, que se ha vuelto oficial, sugiere que los trabajadores esenciales son personas que realizan labores consideradas indispensables para el mantenimiento de la vida cotidiana de la sociedad. Por lo tanto, hacen parte de las estructuras que garantizan la seguridad nacional del país y, por ley, deben asistir a sus locales de empleo. Como "trabajadores esenciales", los latino/as, independientemente de su estatus legal, han sido los más afectados por esta pandemia, ya que son los que han tenido que salir a trabajar para hacer todo tipo de servicios, con o sin equipo de protección. Esta nueva categoría oficial de "trabajadores esenciales" incluye muchos de los empleos realizados por latino/as. Incluye por ejemplo a todas "las personas que limpian los pisos, que lavan la ropa, que sirven comida rápida, que cosechan los cultivos, que trabajan en las plantas de carne, además de los trabajadores de hogares de ancianos, las enfermeras y auxiliares de enfermería que trabajan en hospitales o que cuidan a los descapacitados y, en general, a personas que mantienen servicios esenciales en las ciudades grandes y chicas, así como en los

The Price of Being Essential: Latino Essential Workers bear brunt of Corona virus. Los Angeles Times. May 17, 2020. https://www.latimes.com/california/story/2020-0517/latino-essential-workers-coronavirus pueblos y aldeas para que el resto de la población pueda quedarse en casa." 2

En este contexto vale preguntar: ¿Cuán esenciales son las personas a quienes, por otra parte, también se les considera como desechables?

En el contexto de las altas tasas de desempleo y el empeoramiento de la situación económica en los Estados Unidos hoy, un trabajador nos contesta de esta manera:

Con la recesión que se avecina, "todos estarán en la economía de los cachuelos ( "gig economy"; "trabajito" "o bico"). ... no se trata sólo de las minorías sistemáticamente empobrecidas, ni de los inmigrantes. Comienza con ellos, [con] nosotros; yo mismo [soy\} mexicano", nos dice. "Y ahora estamos viendo ... que más gente de la clase media está siendo despedida de nuevo, igual que la última vez [en 2008] ... nos llaman trabajadores esenciales ... parece más que somos prescindibles. Le garantizo que hay algunos memorandos a puerta cerrada que dicen ... "Cuando mueran, simplemente los reemplazaremos." Es desgarrador ... 3

Al abordar la experiencia de los latino/as estadounidenses del COVID-19, es importante afirmar que, como con cualquier otro problema en los Estados

\footnotetext{
Matthew Telles. Essential or Expendable? Gig Workers at Instacart \& Grocery Stores Demand Safety Gear \& Hazard Pay. Interview. $\begin{array}{lll}\text { Democracynow.org. April 20, } 2020 . & .\end{array}$ https://www.democracynow.org/2020/4/20/matthew telles_instacart_gig_workers
} 
Unidos, las disparidades raciales han moldeado el desarrollo y los resultados de esta pandemia en todo el país. ${ }^{4} \quad$ A principios de abril,

\begin{abstract}
"los informes de Louisiana mostraron que los afroamericanos representaron el 70 por ciento de las muertes, a pesar de ser el 30 por ciento de la población. También a principios de abril, las personas latinas constituían el 33 por ciento de las muertes por coronavirus en la ciudad de Nueva York. Los afroamericanos en Chicago están muriendo a un ritmo seis veces mayor que el de sus vecinos blancos. En Nuevo México, los Indígenas americanos son menos del 10 por ciento de la población, pero tienen un tercio de los casos confirmados de coronavirus. Y los asiático-estadounidenses tienen un poco más de probabilidades de morir por el virus que sus homólogos blancos." ${ }^{5}$
\end{abstract}

Parte de la razón de las altas tasas de mortalidad es el hecho de que los trabajadores esenciales, independientemente de su raza, no tIenen

\footnotetext{
${ }^{4}$ Sin duda, la desigualdad económica y los problemas que suscita como la distribución de la riqueza, el empleo, el acceso a la atención médica y la educación, son temas esenciales en esta discusión. Pero en los Estados Unidos, esa discusión también está determinada por el hecho que "las familias blancas tienen sustancialmente más riqueza que las familias negras e hispanas". De hecho, es importante tener en cuenta la dimensión racial de las disparidades de riqueza en los Estados Unidos. Una encuesta, realizada por el Centro para el Crecimiento Equitativo de Washington, demuestra que "La riqueza media para las familias en las que el encuestado era blanco y no hispano o latino en 2016 fue de $\$ 171,000$. La riqueza media para las familias en las que el encuestado era negro o afroamericano y no hispano o latino era de $\$ 17,000$, y la riqueza media para las familias en las que el encuestado era hispano o latino era de $\$ 21,000$. La riqueza media para todas las demás familias fue de $\$ 65,000$. " Por otra parte, el acceso a la atención médica, independientemente de la calidad, particularmente en un contexto privatizado como
}

acceso al equipo de protección necesario para mantenerlos a salvo. Pero sin duda gran parte de la alta mortalidad también se debe al numero elevado de personas de color, especialmente entre afroamericanos y latino/as cuyos empleos requieren que trabajen en espacios cerrados, ya sea dentro de fábricas o en edificios de oficinas, etc. En el caso de los latino/as, solo el $16 \%$ podía trabajar en casa, al contrario que otros grupos. Como resultado, desde mediados de marzo, cuando esos espacios tuvieron que cerrar, millones de latino/as que no eran "trabajadores esenciales" simplemente fueron despedidos de sus empleos. A fines de mayo, según la Oficina de Estadísticas Laborales, 41 millones de estadounidenses estaban desempleados, y eso a pesar de que desde abril, varios estados ya habían comenzado a abrir sus economías. De ese número, $17.6 \%$ eran latino/as. ${ }^{6}$

Estados Unidos, está determinado por la capacidad de las personas para pagar la cobertura. Los más afectados por COVID 19 en la sociedad estadounidense han sido personas de color, y esto a pesar del hecho de que una pandemia supuestamente no conoce raza, etnia, clase ni distinciones generacionales. Cf. https://equitablegrowth.org/the-distribution-of-wealthin-the-united-states-and-implications-for-a-net-worthtax/)

5 Cynthia Greenlee. Documenting Disparity: The Challenges of Collecting Racial Data on Coronavirus. Colorlines, APR 29, 2020 1:45PM ET. https://www.colorlines.com/articles/documentingdisparity-challenges-collecting-racial-data-coronavirus

6 UnidosUS. "Latino Unemployment Rate Remains the Highest at 17.6\%." Latinos Job Reports. June, 2020. https://www.unidosus.org/aboutus/media/press/releases/06520-UnidosUS-ReleasesMay-Jobs-Report 
Soy muy consciente de que escribo este ensayo a mediados del mes de junio del 2020 sabiendo que ya han fallecido 114,000 personas a causa del coronavirus en Estados Unidos. El número de contagios en este país ya superó los 2,043,000 de casos. Sin duda, la situación aquí es terrible; las muertes, el hambre, el miedo, la desesperación se ve y se siente por todas partes. El país está sin líderes at nivel nacional $-\mathrm{y}$ creo que es importante recalcar ese punto. También es importante subrayar que en este país desde un comienzo ha habido una tensión muy grande entre el gobierno federal y los gobiernos estatales. El resultado es que no hay liderazgo nacional y cada estado maneja la pandemia a su manera. La consecuencia es que esa "política" de cadauno-por-sí-solo que continua a ser implementada de manera espontánea en los 50 estados del país, nos ha llevado a un nivel peligroso en el manejo de la enfermedad, ya que no permite que se desarrolle una política de salud pública a nivel nacional, parecida a la que se ha implementado en otros países. Al contrario, Trump se ha aprovechado de la tensión histórica que existe entre el gobierno federal y los estados, para impulsar sus propios fines e intereses electorales, y no cesa de enfrentar un estado contra el otro, a veces con resultados desastrosos para la salud pública de los ciudadanos de este país ...
Por otra parte, también es importante reconocer que, a pesar de toda la miseria, el sufrimiento, y la desesperación que mucha gente, y especialmente, la gente de color está viviendo, hay también mientras tanto pequeños rayos de luz. En otras palabras, hay un cambio definitivo en el significado de la pertenencia, y tanto la ayuda mutua como la solidaridad están creciendo a nivel local en el país. Sin duda, el COVID-19 ha expuesto a la luz, lo profundo que son las disparidades raciales en este país.

En estos últimos meses esas disparidades han empeorado y también son más evidentes por el hecho de que esta pandemia se está desarrollando bajo un presidente que como hemos visto, tiene la intención explícita de utilizar abiertamente la supremacía blanca para destruir los valores, las tradiciones, instituciones nacionales $\mathrm{y}$, sobretodo, la vida, las esperanzas y las expectativas, particularmente de las personas de color en los Estados Unidos. Al mismo tiempo, la brecha entre lo que el pueblo estadounidense realmente quiere y lo que el liderazgo del país en Washington les ofrece, parece estar creciendo exponencialmente. Es una fisura que crece con cada día que pasa y expone tanto las debilidades como la fortaleza de las instituciones de este país que hasta hoy se declara estar fundamentado en un gobierno que es de, para y por el pueblo. De hecho, si hay algo positivo que esta pandemia trae --y 
que el COVID-19 de alguna manera ha ayudado a impulsar al primer plano de la conciencia estadounidense ya sea en las protestas multirraciales, o en la profundidad con que el movimiento Black Lives Matter resuena hoy en día en esta sociedad-- es que el COVID-19 ha permitido a las personas que han sido tratadas como prescindibles $\mathrm{y}$ desechables, probar la veracidad de la propuesta de que Estados Unidos tiene un gobierno que es de, para y por el pueblo. Y podemos verlo más claramente en estas semanas desde la muerte de George Floyd y en las protestas continuas contra el asesinato de hombres afroamericanos por la policía en el país entero y la determinación de los gobiernos locales y estatales de responder a las demandas del movimiento Black Lives Matter.

De hecho, a nivel federal, debido a que Trump ha abdicado literalmente de sus responsabilidades y liderazgo tradicionales durante una crisis nacional, los líderes de los gobiernos locales y estatales están trabajando con organizaciones de base en varias partes del país para abordar la pandemia y salvar vidas en medio del caos. No me refiero solo a la falta de liderazgo y acción en términos de organizar pruebas, equipos de protección, de ventiladores, etc. Estoy hablando más sobre la naturaleza sin precedentes de esta epidemia que sorprendió a personas de todo el mundo.

En este país, la falta de cualquier tipo de políticas de salud pública y otras formas de asistencia del gobierno federal en muchos casos ha llevado al desarrollo de nuevas relaciones a menudo con la ayuda de organizaciones locales y de base.

Unos ejemplos ilustran la paradoja de los latino/as como trabajadores esenciales que a la vez son tratados como prescindibles y desechables. Esto también implica indagar cómo las familias latinas están lidiando con la pandemia y qué nos dicen sus experiencias sobre lo que está sucediendo en el país en general.

Comienzo primero con los trabajadores agrícolas, principalmente los trabajadores agrícolas en California, ya que la gran mayoría de latino/as considerados como trabajadores esenciales trabajan en ese ramo de la economía. Y luego pasaré a unos ejemplos de lo que está sucediendo con la comunidad latina en la ciudad de Nueva York, particularmente en lo que estoy denominando la "economía de los cachuelitos" (o trabajitos) -y que incluye trabajadores que hacen compras de comestibles para los otros, por ejemplo, a través de aplicaciones como Instagram o Amazon; que son choferes de Uber, que trabajan en supermercados, etc. Escogí hablar sobre Nueva York en parte porque ha sido durante muchos meses el epicentro del COVID 19, y me parece importante documentar unos ejemplos de lo que los latino/as están haciendo para enfrentar su vida diaria. 
Comienzo entonces con los trabajadores agrícolas de California. Gran parte de esta información la obtuve de un panel reciente en la Universidad Estatal de California, en Sacramento, que reunió a representantes de organizaciones de base, como el grupo Lideres Campesinas y la Fundación de Asistencia Legal Rural, el Consejo Laboral para el Avance Latinoamericano, así como a activistas y sindicalistas. $^{7}$ El tema fue el trabajo esencial de los trabajadores agrícolas durante la pandemia. El estado de California es la quinta economía mas importante a nivel mundial. La industria agrícola forma gran parte de ella. Los trabajadores agrícolas son responsables por la producción de alimentos no solo para todo el estado de California sino también para toda la nación, así como para las exportaciones de alimentos de California. Según Manuel Barajas, profesor de la Universidad Estatal de California, Sacramento, de los 2.5 millones de trabajadores agrícolas en los Estados Unidos, aproximadamente la mitad son indocumentados y tienen cero protección de salario. En tiempos normales, muchos de ellos viven con temor continuo -y algunos, como también señala Barajas, con cólera

\footnotetext{
7 Manuel Barajas (panel organizer) Introduction. Essential Labor: Farmworkers during the Pandemic. Center on Race, Immigration and Social Justice
}

continua- por la constante posibilidad de que los van a deportar.

Cuando la pandemia golpeó a los Estados Unidos en febrero y marzo, la retórica sobre el peligro de los inmigrantes para la nación y la economía estaba en su punto más alto. A pesar de la pandemia, ICE, la agencia del Control de Inmigración y de Aduanas, que pertenece al Departamento de Seguridad Nacional continuó sus redadas y alarmas de miedo en comunidades de inmigrantes en todo el país, particularmente en estados demócratas como Nueva York y California que durante mucho tiempo han estado en guerra con Trump. Como señaló Barajas, el gobierno de Estados Unidos continuaba con su retórica en contra de los inmigrantes, a la vez que comenzó a entregar visas temporales $\mathrm{H}-2 \mathrm{~A}$ a trabajadores agrícolas. A pesar de haber "cerrado la frontera" formalmente, el gobierno también se aseguró de que los grandes intereses agrícolas supieran que las visas $\mathrm{H} 2$ A estaban disponibles para la renovación de los trabajadores agrícolas que habían trabajado en los Estados Unidos el año anterior. Hoy en día, estos trabajadores son parte de los 2.5 millones de trabajadores agrícolas en los Estados Unidos que aseguran la continuidad del suministro de alimentos del país, a pesar de trabajar sin acceso a cualquier tipo de equipo de protección.

https://csus.mediasite.com/Mediasite/Play/826eec57e76 04816859b4ed2c7caa09b1d Accessed 6-14-2020. 
Es importante recalcar que a pesar de la gran solidaridad que existe en los EE. UU. para con los trabajadores esenciales, éstos pueden ser, y son despedidos si se quejan. También pueden ser despedidos si no se presentan a trabajar, incluso cuando están enfermos. Esto, por supuesto, no es nuevo, cuando se trata de la experiencia de los trabajadores agrícolas en el país, en particular de los aproximadamente 1.2 millones de trabajadores agrícolas, tanto los indocumentados como aquellos que fueron traídos de México como "trabajadores temporales invitados." (guest workers). Juntos representan entre el $1 \%$ y el $2 \%$ de la fuerza laboral total de los Estados Unidos. La mayoría son mexicanos, entre los cuales hay muchos indígenas, ya sea mixtecos, otomíes, náhuatl, etc. Como también señaló Barajas, en California, el agronegocio es una industria de más de 20 mil millones de dólares, que depende de millones de latino/as entre otros ciudadanos e inmigrantes que ahora también han sido categorizados como "trabajadores esenciales" en todo el país, ya sea porque trabajan en la industria del transporte, en servicios de entregas de supermercados, etc.

Debido a que muchos trabajadores agrícolas son indocumentados, y también porque son trabajadores temporales, la mayoría no reciben ayuda ni protección del gobierno, a pesar de que ahora están categorizados como trabajadores esenciales y reconocidos como tales. En marzo pasado, mientras las corporaciones de agronegocios de la nación obtuvieron un apoyo importante del proyecto de ley de estímulo gubernamental de 2.2 mil millones de dólares, los trabajadores agrícolas no recibieron nada, y su seguridad y salud también se descuidaron por completo. Estaban estresados por no tener trabajo, por la desaparición de sus ahorros de vida debido a COVID-19, por quedarse sin alimentos porque no califican para el seguro de desempleo.

En términos de las condiciones laborales reales, Irene de Barraicua Directora de Relaciones Públicas de la organización Líderes Campesinas señaló que existen políticas que requieren que los contratistas brinden atención preventiva, agua limpia, y baños sanitarios, pero estas políticas nunca se aplicaron incluso antes de COVID-19, mucho menos durante COVID-19. Los trabajadores agrícolas tampoco califican para el seguro médico, por lo que no podrían ir a médicos u hospitales si estuvieran enfermos. La vivienda también es una preocupación pública importante: muchos viven en sus automóviles $\mathrm{y}$ antes por lo general tenían acceso a tiendas donde podían lavarse las manos y ducharse. Pero ahora todo está cerrado.

Dada la importancia de los trabajadores agrícolas, varias organizaciones de base, así como activistas del sindicato United Farmworkers (UFW) entre otras organizaciones, hicieron encuestas. 
Basándose en las recomendaciones de los propios trabajadores, escribieron cartas al gobernador del estado de California, entre otros, instándolos a promulgar nuevas políticas para proteger a los trabajadores agrícolas, en muchos casos por primera vez. Y, como señaló Juanita Ontiveros, representante de la Fundación de Asistencia Rural Legal de California, y de la UFW, los gobernadores respondieron, porque saben que si el COVID-19 destruye la salud de los trabajadores agrícolas, la amenaza no es solo para éstos sino también para el suministro de alimentos. A través de algunas de sus demandas y recomendaciones, podemos discernir un poco las condiciones laborales, y también entender por qué el COVID-19 ha puesto a los trabajadores agrícolas y a todos los trabajadores esenciales en la industria alimentaria en una situación muy difícil: tienen miedo al coronavirus y también tienen miedo de alimentar a la nación. Entre otras, Ontivero, al igual que otros miembros del panel, explicitó que sus demandas incluyen:

- "Extender la licencia por enfermedad a 40 horas o más.

- Eliminar la espera de 90 días para que las personas sean elegibles para días de licencia por enfermedad.

- Eliminar la necesidad de una nota del médico para tomarse unos días de licencia por enfermedad

- Implementar la distancia social en los campos para proteger a los trabajadores y minimizar las infecciones.
- Limpiar y desinfectar las superficies tocadas varias veces al día

- Organizar el acceso fácil a los servicios médicos.

- Proporcionar exámenes de detección, pruebas y tratamiento para la gran mayoría que no tiene atención médica.

- Ofrecer cuidado de niños y más flexibilidad dado que las escuelas están cerradas ..."

\section{Como consecuencia del COVID-19}

en algunas partes de California se ha comenzado a aprobar por primera vez nuevas leyes para abordar éstas y otras demandas --un ejemplo de un cambio que resulta directamente de las demandas $\mathrm{y}$ necesidades de los trabajadores agrícolas y sus partidarios de base, independientemente de su estatus legal.

Por cierto, como bien señalaron los panelistas, los trabajadores agrícolas arriesgan su propia salud para asegurarse de que la nación tenga acceso a frutas $\mathrm{y}$ verduras. Desempeñan un papel esencial en la seguridad alimentaria. $\mathrm{Y}$ aunque las repercusiones de las formas indiferentes anteriores a COVID-19 continúan en muchas partes del país, principalmente entre los conservadores, a nivel de la ciudadanía, también existe una creciente conciencia y gratitud entre la gente común por la importancia del trabajador.

La importancia de los trabajadores esenciales es en realidad algo que está 
siendo reconocido por la mayoría de la población a través del país. Esto se ve claramente en el ejemplo de Nueva York donde todas las noches a las 7 p.m., los siete días de la semana, se escuchan las caceroladas, agradeciendo a los trabajadores esenciales por su trabajo.

El bloqueo de tres meses en la ciudad de Nueva York cambió la ciudad por completo. No había automóviles, no había personas en la calle --conducir en la ciudad era como conducir en un pueblo fantasma. Los únicos vehículos eran las muchas ambulancias, los únicos sonidos de la ciudad, las sirenas siempre penetrantes y sin parar de esas ambulancias. Junto con el silencio de las calles, el vacío total de las aceras, los edificios, cerrados, la falta chocante de seres humanos, de vida, andar por la ciudad daba la sensación de estar dentro de una película de ciencia ficción.

En este contexto, es importante señalar que los latino/as representan aproximadamente el 29\% de la población de la ciudad de Nueva York y para el 6 de abril representaban casi el $34 \%$ de los pacientes que murieron de COVID-19. ${ }^{8}$ Muchos latino/as que trabajaban en industrias que estaban cerradas como la construcción, escuelas, restaurantes, hoteles, quedaron desempleados. Por otro lado, entre los

\footnotetext{
${ }^{8}$ Colin Dwyer. "New York City's Latinx Residents Hit Hardest By Coronavirus Deaths." NPR. April 8, 20201:06
}

trabajadores esenciales en Nueva York, a las personas que trabajaban entregando paquetes se les decía que se presentaran a trabajar con o sin fiebre.

El sistema médico y de salud de la ciudad no fue suficiente para resistir la cantidad de personas que tenía que cuidar. Aproximadamente 315 pacientes infectados con COVID-19 fueron tratados en las tiendas de campaña improvisadas en el Central Park de Nueva York, debido a la falta de camas de hospital en la ciudad.

Hasta hoy, han habido 211 mil casos confirmados en la ciudad de Nueva York, y más de 17,000 muertes. Sin duda las cifras totales son más altas ya que la ciudad solo contó las muertes confirmadas (es decir, muertes en hospitales). Existen numerosas historias de personas que mueren en sus casas. Hay historias de que la ciudad tuvo que traer camiones de refrigeración para depositar a los muertos; historias de la desesperación de las personas que tenían que esperar cuatro a cinco semanas para conseguir una funeraria para enterrar a sus muertos; historias de las 120 fosas comunes que habían sido excavadas hasta abril de 2020 en la isla Hart, una pequeña isla frente a la costa en el Bronx. Allí enterraron personas, muchos quizás eran inmigrantes, cuyos cuerpos no habían sido reclamados; personas que no podían pagar un

https://www.npr.org/2020/04/08/829726964/new-yorkcitys-latinx-residents-hit-hardest-by-coronavirus-deaths 
funeral también fueron enterradas allí, muchas de las cuales eran trabajadores indocumentados, trabajadores esenciales que se mudaron a los Estados Unidos para crear una vida mejor para ellos, y ahora como alguien dijo, "se mudan de la cama del hospital, al camión refrigerado, al incinerador." 9

Las estadísticas realmente no cuentan la historia de la experiencia de los latino/as en absoluto. Las barreras del idioma, por supuesto, explican la falta de información y conocimiento sobre el virus. El estado migratorio definitivamente ha impactado el acceso de las personas y el deseo de buscar atención. Particularmente en los primeros meses y hasta hoy, hay temor entre los indocumentados, que fueron los menos capaces de mantenerse a sí mismos sin salir principalmente era miedo a la agencia ICE de inmigración- hasta que el gobierno federal finalmente acordó un cese temporal de sus acciones contra los inmigrantes. ${ }^{10}$

A la misma vez, también había miedo a COVID-19. Luego vino la ola de desempleo y el temor de no encontrar los medios para poner comida en la mesa, o de

\footnotetext{
${ }^{9}$ Adriana Gallardo and Ariel Goodman. Undocumented New Yorkers Are Essential and Underprotected in COVID's Epicenter. ProPublica, 5-2-2020 https://truthout.org/articles/undocumented-newyorkers-are-essential-and-underprotected-in-covidsepicenter/

10 En Marzo del 2020, el New England Journal of Medicine discutió la situación de los inmigrantes, señalando que Covid-19 ha expuesto debilidades en el sistema de salud de EE. UU.
}

enviar el dinero necesario para los seres queridos que han dejado atrás, para sobrevivir a los toques de queda en su lugar de nacimiento. ¿Cómo se logra una distancia social saludable entre las familias multigeneracionales obligadas a vivir en espacios pequeños, a veces solo de una o dos habitaciones? Aunque muchos latino/as indocumentados han vivido en los EE. UU. y Nueva York durante más de una década, contribuyendo todos los días a la vida y al desarrollo de la ciudad, en cualquier trabajo de baja remuneración que puedan encontrar, durante la pandemia no pudieron acceder a ningún tipo de apoyo del gobierno federal. A pesar de ofrecer algunos servicios, más de 2 millones de familias fueron excluidas de la ayuda económica de los gobiernos federal, estatal o de la ciudad, por ser indocumentados, lo que llevó a la movilización de varios grupos en el estado en el mes de abril. ${ }^{11}$ En algunos casos, la ciudad de Nueva York no les ayudaría ni siquiera a financiar el entierro de sus muertos. Esto se debe a que la ciudad requiere números de seguridad social tanto para el fallecido como para la persona que

https://www.nejm.org/doi/full/10.1056/NEJMp20059 $\underline{53}$

11 Jason Grant. "Cuomo Urged to Provide COVID-19 Economic Relief to Undocumented Workers". April 18, 2020. https://www.law.com/newyorklawjournal/2020/04/18 /cuomo-urged-to-provide-covid-19-economic-reliefto-undocumentedworkers/?slreturn $=20200620110026$ 
solicita fondos para proporcionar asistencia para el entierro. Además, las familias de los 417 mexicanos que murieron a causa de la pandemia, y que querían enviar los cuerpos de sus familiares a las madres, hermanos, hermanas o hijos del fallecido tampoco pudieron hacerlo debido al propio gobierno de México, que permitió que los cuerpos fueran devueltos a México para su entierro solo si habían sido incinerados.

La desconfianza del gobierno federal, estatal o municipal- es muy alta entre los inmigrantes de Nueva York, y con razón. Poco antes de que el COVID-19 llegara al noreste de los EE. UU., Trump ya había aprobado el proyecto de ley de "carga pública" por la que quien intentara obtener servicios sociales, incluida la salud y el bienestar del estado, ya no sería elegible para postular a la obtención de la residencia estadounidense -(conocida como el "Green Card") ni a la ciudadanía. Aunque la administración Trump acordó posponer el proyecto de ley de carga pública, muchos latino/as se niegan a pedir asistencia médica o alimentaria o ayuda alguna por miedo a descalificarse para obtener la residencia permanente en el país.

Por otra parte, en la ausencia de la asistencia del gobierno, yo he sido testigo de muestras de solidaridad de apoyo mutuo y de humanidad, que también hace parte importante del sistema de valores estadounidense. Ya sea como señalé en el caso de los trabajadores agrícolas, o en el caso de los indocumentados, o de todos aquellos que necesitan asistencia de alguna manera en la ciudad de Nueva York y en otros lugares, organizaciones de base similares a las que sirven a los trabajadores agrícolas, o asociaciones y otros grupos del país de origen, han trabajado durante años para mitigar las dificultades en la vida de las personas. Hoy, bajo el gobierno de Trump, han intensificado sus esfuerzos para responder a la nueva situación COVID-19.

Ahora escriben cartas a los gobernadores con recomendaciones que en muchos casos son muy bien recibidas por funcionarios locales abrumados. Están ayudando a diseñar nuevas políticas y a trabajar junto con los gobiernos y las agencias locales para garantizar que los necesitados reciban ayuda y apoyo ya sea con alimentos, o con políticas que permitan el atraso o la exoneración del pago de alquiler. En Los Ángeles, las madres y los niños sin hogar se apoderan de las casas abandonadas para que ellos también puedan distanciarse socialmente y protegerse de la enfermedad. A instancias de estas organizaciones, las cárceles comienzan a liberar a internos que no son violentos para reducir la propagación de la enfermedad. Estas organizaciones también están utilizando sus sistemas de apoyo 
desarrollados desde hace mucho tiempo para difundir información y alimentar a los hambrientos y apoyar a las comunidades de todas las formas posibles.

Recientemente, una joven nos contó que un día conducía con su padre en Queens, un condado de la ciudad Nueva York, que junto con el Bronx son los más afectados por el Covid-19. Al ver filas enormes de personas esperando fuera de la Asociación Ecuatoriana para recibir apoyo de alimentos y otras necesidades, la joven, sabiendo que muchos niños de Nueva York dependen de las escuelas públicas de la ciudad que ahora están cerradas para alimentarse, se fue a su casa y creó una página web de GoFundMe. En pocos días, recaudó suficiente dinero para permitir que su familia pudiese cocinar y llevar suficiente comida para alimentar a 300 familias.

Está claro que Trump y la cultura política corporativa en Washington que lo apoya no están allí para gobernar. Al mismo tiempo, estas organizaciones de base y ciudadanos individuales se están movilizando y mostrando su apoyo a los más vulnerables. Estas muestras de solidaridad y apoyo se ven en todo el país, en ciudades grandes y pequeñas. Es verdad que no representan necesariamente el gran cambio necesario. Sin embargo, al igual que las continuas y poderosas protestas de Black

12 Citado por Joe Hansen. "The Moral Test of Government." Huffington Post. 09/03/2012 02:06 pm ET Updated Nov 03, 2012.
Lives Matter de las que hemos sido testigos desde el asesinato de George Floyd por parte de la policía el 25 de mayo de 2020, hay un nuevo sentido de ciudadanía y participación en el aire, que algún día podría volverse lo suficientemente fuerte como para desafiar el claro esfuerzo que está haciendo Trump para destruir las instituciones de los Estados Unidos.

Quiero terminar con una reflexión que es el resultado de un comentario del vicepresidente Hubert Humphrey (19651977) quien en 1977 declaró lo siguiente acerca de cómo el gobierno es un reflejo de su "trato a los miembros más débiles de la sociedad". Humphrey dijo:

La prueba moral del gobierno es cómo ese gobierno trata a los que están en los albores de la vida, los niños; los que están en el ocaso de la vida, los ancianos; y los que están en las sombras de la vida, los enfermos, los necesitados y los discapacitados. ${ }^{12}$

Vemos que el gobierno actual no los trata bien... lo que sí vemos también es que la humanidad y los valores del pueblo estadounidense en las calles del país están comenzando a florecer -ojalá que siga floreciendo....

https://www.huffpost.com/entry/the-moral-test-ofgovernm_b_1847379 


\section{Referências}

Suzanne Oboler. Extraños Desechables: raza e inmigración en la era de la globalización" InterDisciplina: 2(4) Sept/Dec, 2014; pp 75-96

The Price of Being Essential: Latino Essential Workers bear brunt of Corona virus. Los Angeles Times. May 17, 2020.

https://www.latimes.com/california/story/2020-0517/latino-essential-workers-coronavirus

Matthew Telles. Essential or Expendable? Gig Workers at Instacart \& Grocery Stores Demand Safety Gear \& Hazard Pay. Interview.

Democracynow.org. April 20, 2020.

https://www.democracynow.org/2020/4/20/matthe w_telles_instacart_gig_workers

Cynthia Greenlee. Documenting Disparity: The Challenges of Collecting Racial Data on

Coronavirus. Colorlines

APR 29, 2020 1:45PM ET.

https://www.colorlines.com/articles/documentingdisparity-challenges-collecting-racial-datacoronavirus

UnidosUS. "Latino Unemployment Rate Remains the Highest at 17.6\%." Latinos Job Reports. June, 2020.

https://www.unidosus.org/aboutus/media/press/releases/06520-UnidosUS-ReleasesMay-Jobs-Report

Manuel Barajas (panel organizer) Introduction. Essential Labor: Farmworkers during the Pandemic. Center on Race, Immigration and Social Justice . https://csus.mediasite.com/Mediasite/Play/826eec5 7e7604816859b4ed2c7caa09b1d

Accessed 6-14-2020.

Colin Dwyer. "New York City's Latinx Residents Hit Hardest By Coronavirus Deaths." NPR. April 8, 20201:06 PM ET

https://www.npr.org/2020/04/08/829726964/newyork-citys-latinx-residents-hit-hardest-bycoronavirus-deaths

Adriana Gallardo and Ariel Goodman.

Undocumented New Yorkers Are Essential and Underprotected in COVID's Epicenter. ProPublica, 5-2-2020

https://truthout.org/articles/undocumented-newyorkers-are-essential-and-underprotected-in-covidsepicenter/

${ }^{1}$ En Marzo del 2020, el New England Journal of Medicine discutió la situación de los inmigrantes, señalando que Covid-19 ha expuesto debilidades en el sistema de salud de EE. UU. https://www.nejm.org/doi/full/10.1056/NEJMp200 5953

Jason Grant. "Cuomo Urged to Provide COVID-19 Economic Relief to Undocumented Workers". April 18, 2020.

https://www.law.com/newyorklawjournal/2020/04/ 18/cuomo-urged-to-provide-covid-19-economicrelief-to-undocumentedworkers/?slreturn=20200620110026

"The Moral Test of Government." Huffington Post. 09/03/2012 02:06 pm ET Updated Nov 03, 2012. https://www.huffpost.com/entry/the-moral-test-ofgovernm_b_1847379 